\title{
Human Upper Limb Mass-Inertial Characteristics via Computer Modelling
}

\author{
Gergana Nikolova, Daniel Dantchev, Alexander Kazakoff \\ Institute of Mechanics - Bulgarian Academy of Sciences \\ Acad. G. Bonchev Str., Block 4, 1113 Sofia, Bulgaria \\ gergana@imbm.bas.bg,daniel@imbm.bas.bg, alex_kazakoff@yahoo.co.uk
}

\begin{abstract}
We present a new modified biomechanical geometrical model of human upper limb, with the help of three-dimensional geometrical mathematical modelling - 3D (GMM). This model represents a modification of the ones suggested in $[1,2,3,4]$ and is based on our own anthropometric measurements of 50 men aged between 30-40 years. Using the model, we calculate the volume and mass, the center of mass and the principal moments of inertia for the human body upper limb for two basic sub-cases: $i$ ) determination of these parameters for the separate parts of the upper limb: upper arm, lower arm and hand and ii) for the human upper limb as a whole. For the last we used the computer realization of the model. In order to validate the accuracy of the program we have performed a detailed comparison of the numerical results obtained within the program with the numerical evaluation of the analytical expressions that we have derived for the corresponding quantities. We observed an excellent agreement between the two approaches. Finally, we also present a comparison between the results obtained within our model with data for other Caucasian reported in literature. We observe an overall good agreement among all of them with the data gained for Bulgarian males. The proposed model is oriented to application in medicine (orthopedics and traumatology) and engineering when designing devices aimed to help disabled individuals. It can predict data for the inertial parameters of a given male individual provided the corresponding easily measurable geometrical data for this individual are known.
\end{abstract}

Keywords: Biomechanics, mass moments of inertia, human upper limb model, three-dimensional geometrical mathematical modelling - 3D (GMM), manipulation system, body segment parameters, mass-inertial characteristics, mass properties

\section{Introduction}

The investigation on the human body geometric and mass-inertial characteristics is of key importance in human motion analysis. In our everyday activities - when working, driving a vehicle, even when taking a meal, or even drinking a water and not to speak about all possible sport activities the use of the limbs is crucial. The last is, obviously, to be taken into account when one needs to design a devise for supporting an impaired person in any of these activities and to provide, as good as possible, the quality of live for such an individual. We are going to study the mass-inertial characteristics of a male person with the help of mathematical and computer modeling. When doing that, one has to resolve a set of problems. They include: 1) the proper segmentation of the limb; 2) the choice of the anthropometric points used to defined these segments; 3) the set of geometrical data characterizing the so defined segments; 4) the choice of geometrical figures with the help of which the segments are to be modeled; 5) determination of the needed geometrical characteristic pertinent for this geometrical bodies based on the measured data; 6) derivation of mathematical expressions for any of the characteristics of interest; 7) numerical evaluation of these expressions using experimentally available data for the additional information needed, say, the density of the corresponding segment; 8) computer realization of the mathematical model in the framework of a suitable software package; 9) validation of the computer model via comparison of the data obtained from the computer model with the data obtained from the analytical expressions; 10) obtaining data on the basis of the computer model for situations in which analytical results are missing or is elaborative to be derived; 11) comparison of the so derived data with the ones reported previously, when available, in the literature for persons belonging to the same or similar type.

In the current article we have realized the above program of points for the upper limb of an average Bulgarian male. 
One of the earliest studies of mass, volume and center of mass of male cadavers are those of [5, 6]. In the 60-s and 70$\mathrm{s}$ a number of studies have reported anthropometric and mass-inertial of the segments of the human body of elderly male cadavers [7, 8, 9]. Different methods for investigation of body segment mass-inertial parameters obtained for living male individuals have been used: immersion and cast method [10], gamma mass scanning [11], geometrical modeling [12, 13]. The approach utilized in the current study is based on geometrical modeling.

\section{The Model and the Procedure}

Let us now explain in a bit more details what we have actually done in order to achieve the goals outlined above aimed to determine the mass inertial parameters of the upper limb of the average Bulgarian male.

We start from the model proposed [1] in of the average Bulgarian man. In the current article, however, we improve the way how one models there the upper arm and the lower arm. Instead of considering them of using frustums of a cone, as in [1], we represent these parts of the upper limb with versions of right elliptical stadium solids (see Fig. 1). The modeling of hand (fist) remains the same as in $[1,2,3]$. Let us immediately stress that one of the consequences of modelling the upper and lower segments of the upper limb via right elliptical stadium solids is the lack of the "left-right" symmetry for the inertial moments of these segments. The last symmetry was preserved in [1] and is usually also present in most of the geometrical models of the human body we are aware about. In [1] the main part of the geometrical data needed to determine the geometrical parameters of the segments of the body is taken from a detailed representative anthropological investigation of the Bulgarian population [14] where the authors measured a total of 2435 males. Unfortunately, the data collected does not include all the data needed to model the upper and the lower arm as right elliptical solids. For that reason, we made our own complementary anthropometric measurements of these segments on additional 50 Bulgarian males.

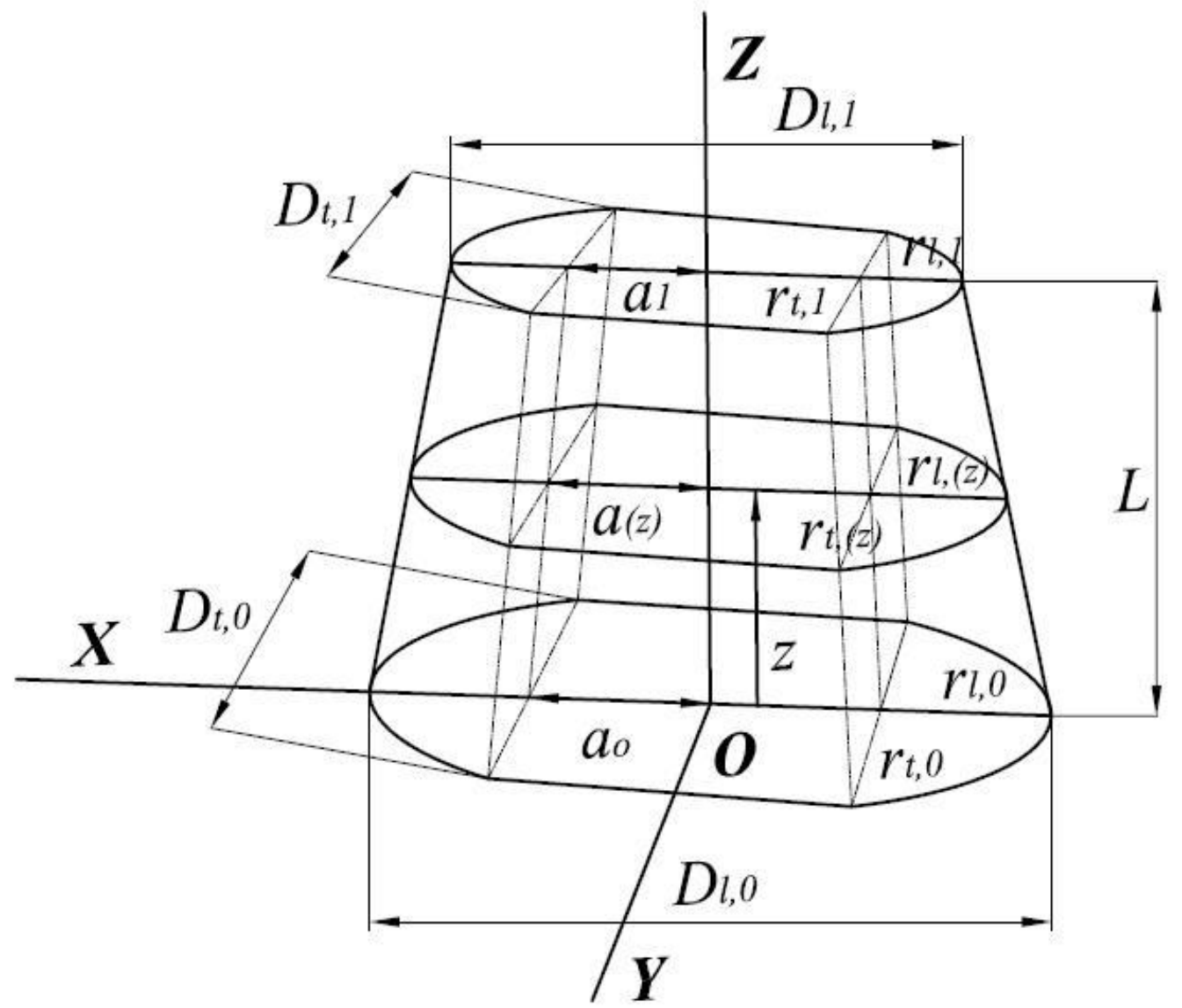

Fig. 1: A right elliptical stadium solid. 


\subsection{Description of the Data}

In the anthropometric measurements, which we performed, data for $D_{l}, D_{t}$ and $L_{c i r}$ (see Fig. 1) have been collected both for the upper and for the lower arm.

- For the upper arm, that extends between acromion-radiale, we measured the circumference across epicondyles $\left(L_{c i r}\right)$, the epicondilar diameter of humerus $\left(D_{l, 0}\right)$, the diameter perpendicular to humerus $\left(D t,{ }_{0}\right)$, as well as the axillary circumference at the proximal end. From these data the values of $a_{0}, a_{l,} r_{l, 0}, r_{l, l}, r_{y, 0}$ and $r_{t, l}$ can be easily inferred: $r_{t}$ directly follows from $r_{t}=D_{t} / 2, r_{l}$ can be determined by numerically solving the equation

$$
L_{c i r}-2 D_{l}=4 r_{l}\left[E\left(\sqrt{1-\frac{r_{t}^{2}}{r_{l}^{2}}}\right)-1\right]
$$

using $r_{t}$ from above, and, finally, one has for $a=\left(D_{l}-2 r_{l}\right) / 2$. The vertical lengths of the upper arm, as well as of all the other segments $(L)$ are taken from [14].

- The lower arm is taken to be, as usual, between the points radiale-stylion. Obviously, the necessity of smooth tailoring demands that the parameters for its proximal end are the same as the above-mentioned dimensions for the distal end of the upper arm. For the wrist (distal end) of lower arm (see Fig. 1), but turned upside down), we measured breadth of radio-ulnar joint $\left(D_{l, 1}\right)$, the thickness in the middle of the radio-ulnar joint perpendicular to its breadth $\left(D t_{, 1}\right)$ and the radioulnar joint circumference $\left(L_{c i r}\right)$.

The average data for the directly measured independent geometrical parameters $D_{l}, D t$ and Lcir described above can be found in [3]. With the data measured for $D_{l}, D t$ and Lcir, and having in mind the analytical properties of the stadium solid, as explained above, one can determine the values of the parameters $a, r_{l}$ and $r_{t}$ characterizing the corresponding segments - the upper and the lower arm, correspondingly. The values, obtained in this way, are reported in Table 1 . Let us remind that, as reported in [1, 2, 3], the height and weight of the average man are $1.71 . \mathrm{m}$ and $77.7 \mathrm{~kg}$. In Table 1 , in additions, the lengths $L$ (in $\mathrm{cm}$ ) of the segments according to [14], also the densities $\rho$ (in $\mathrm{kg} / \mathrm{m}^{3}$ ) of the segments according to [15] are given.

Table 1: Calculated parameters $(\mathrm{cm})$, lengths and densities $\left(\mathrm{kg} / \mathrm{m}^{3}\right)$ for males.

\begin{tabular}{|l|l|l|l|l|l|l|l|l|}
\hline \multirow{2}{*}{ SEGMENT } & \multicolumn{7}{|c|}{ P R A M E T E R (see ref. [3]) } \\
\cline { 2 - 10 } & $a_{\mathrm{o}}$ & $r_{l, 0}$ & $r_{t, 0}$ & $\mathrm{a}_{1}$ & $r_{l, 1}$ & $r_{t, 1}$ & $L$ & $\rho$ \\
\hline UPPER ARM & 3.7 & 0.3 & 3.0 & 4.5 & 0.3 & 3.7 & 30.9 & 1053 \\
\hline LOWER ARM & 3.7 & 0.3 & 3.0 & 2.5 & 0.2 & 1.7 & 24.7 & 1100 \\
\hline SPHERE & & & $\mathrm{R}_{\mathrm{HA}}=4.7$ & & & & & \\
\hline
\end{tabular}

\subsection{Comments on the Procedure}

Using the original experimental data measured and the analytical properties of the solid bodies involved in modelling the segments of the human body, see, e.g., equations (2)-(4), we calculate the mass-inertial characteristics of the average Bulgarian man. We do that for any segment of the upper limb. We have derived analytical expression for the volume of the segments, their mass, the coordinates of the center of mass, and the principal moments of inertia. For simplicity of the exposition we avoid presenting all these quite lengthy expressions here. We have restricted ourselves to presenting the expressions for the principal moments of inertia for the upper arm and lower arm. The moments can be determined using equations (2)-(4). 


$$
\begin{aligned}
& \mathrm{I}_{X X} \\
& =\frac{1}{240} \mathrm{~L} \rho\left\{-\frac{10 L^{2}\left[\pi\left(r_{l, 0}+r_{l, 1}\right) r_{t, 0}+\pi\left(r_{l, 0}+3 r_{l, 1}\right) r_{t, 1}+4 a_{0}\left(r_{t, 0}+r_{t, 1}\right)+4 a_{1}\left(r_{t, 0}+3 r_{t, 1}\right)\right]^{2}}{r_{t, 0}\left[8 a_{0}+4 a_{1}+\pi\left(2 r_{l, 0}+r_{l, 1}\right)\right]+r_{t, 1}\left[4 a_{0}+8 a_{1}+\pi\left(r_{l, 0}+2 r_{l, 1}\right)\right]}\right. \\
& +16 a_{0}\left[4 r_{t, 0}^{3}+3 r_{t, 0}^{2} r_{t, 1}+2 r_{t, 0} r_{t, 1}^{2}+r_{t, 1}^{3}+L^{2}\left(2 r_{t, 0}+3 r_{t, 1}\right)\right] \\
& +16 a_{1}\left[r_{t, 0}^{3}+2 r_{t, 0}^{2} r_{t, 1}+3 r_{t, 0} r_{t, 1}^{2}+4 r_{t, 1}^{3}+3 L^{2}\left(r_{t, 0}+4 r_{t, 1}\right)\right] \\
& +\pi\left[\left(3\left[\left(4 r_{l, 0}+r_{l, 1}\right) r_{t, 0}^{3}+\left(3 r_{l, 0}+2 r_{l, 1}\right) r_{t, 0}^{2} r_{t, 1}\right)+\left(2 r_{l, 0}+3 r_{l, 1}\right) r_{t, 0} r_{t, 1}^{2}+\left(r_{l, 0}+4 r_{l, 1}\right) r_{t, 1}^{3}\right]\right. \\
& \left.\left.+4 L^{2}\left(r_{l, 0}\left(2 r_{t, 0}+3 r_{t, 1}\right)+3 r_{l, 1}\left(r_{t, 0}+4 r_{t, 1}\right)\right)\right]\right\} ;
\end{aligned}
$$

For the hand the expressions needed have been already derived and presented in [1].

$$
\begin{aligned}
& I_{Y Y} \\
& =\frac{1}{720} L \rho\left\{48 a_{0}^{3}\left(4 r_{t, 0}+r_{t, 1}\right)+48 a_{1}^{3}\left(r_{t, 0}+4 r_{t, 1}\right)\right. \\
& +12 a_{0}^{2}\left\{3\left[4 a_{1}+\pi\left(4 r_{l, 0}+r_{l, 1}\right)\right] r_{t, 0}+\left(8 a_{1}+3 \pi r_{l, 0}+2 \pi r_{l, 1}\right) r_{t, 1}\right\} \\
& -30 L^{2} \frac{\left[\left(\pi\left(r_{l, 0}+r_{l, 1}\right) r_{t, 0}+\pi\left(r_{l, 0}+3 r_{l, 1}\right) r_{t, 1}+4 a_{0}\left(r_{t, 0}+r_{t, 1}\right)+4 a_{1}\left(r_{t, 0}+3 r_{t, 1}\right)\right]^{2}\right.}{\left[8 a_{0}+4 a_{1}+\pi\left(2 r_{l, 0}+r_{l, 1}\right)\right] r_{t, 0}+\left[4 a_{0}+8 a_{1}+\pi\left(r_{l, 0}+2 r_{l, 1}\right)\right] r_{t, 1}} \\
& +12 a_{1}^{2} \pi\left[r_{l, 0}\left(2 r_{t, 0}+3 r_{t, 1}\right)+3 r_{l, 1}\left(r_{t, 0}+4 r_{t, 1}\right)\right] \\
& +16 a_{1}\left[4 r_{l, 0} r_{l, 1}\left(2 r_{t, 0}+3 r_{t, 1}\right)+9 L^{2}\left(r_{t, 0}+4 r_{t, 1}\right)+6 r_{l, 1}^{2}\left(r_{t, 0}+4 r_{t, 1}\right)+r_{l, 0}^{2}\left(6 r_{t, 0}+4 r_{t, 1}\right)\right] \\
& +8 a_{0}\left[8\left(6 r_{l, 0}^{2}+3 r_{l, 0} r_{l, 1}+r_{l, 1}^{2}\right) r_{t, 0}+4\left(3 r_{l, 0}^{2}+4 r_{l, 0} r_{l, 1}+3 r_{l, 1}^{2}\right) r_{t, 1}+6 a_{, 1}^{2}\left(2 r_{t, 0}+3 r_{t, 1}\right)\right. \\
& \left.+6 L^{2}\left(2 r_{t, 0}+3 r_{t, 1}\right)+3 \pi a_{1}\left(3 r_{l, 0} r_{t, 0}+2 r_{l, 1} r_{t, 0}+2 r_{l, 0} r_{t, 1}+3 r_{l, 1} r_{t, 1}\right)\right] \\
& +3 \pi\left\{3 r_{l, 0}^{3}\left(4 r_{t, 0}+r_{t, 1}\right)+3 r_{l, 0} r_{l, 1}^{2}\left(2 r_{t, 0}+3 r_{t, 1}\right)+3 r_{l, 1}^{3}\left(r_{t, 0}+4 r_{t, 1}\right)+r_{l, 1} r_{l, 0}^{2}\left(9 r_{t, 0}+6 r_{t, 1}\right)\right. \\
& \left.\left.+4 L^{2}\left[r_{l, 0}\left(2 r_{t, 0}+3 r_{t, 1}\right)+3 r_{l, 1}\left(r_{t, 0}+4 r_{t, 1}\right)\right]\right\}\right\} \\
& I_{z z}=\frac{1}{720} L \rho\left\{48 a_{0}^{3}\left(4 r_{t, 0}+r_{t, 1}\right)+48 a_{1}^{3}\left(r_{t, 0}+4 r_{t, 1}\right)\right. \\
& +12 a_{0}^{2}\left\{3\left[\left(4 a_{1}+\pi\left(4 r_{l, 0}+r_{l, 1}\right)\right] r_{t, 0}+\left(8 a_{1}+3 \pi r_{l, 0}+2 \pi r_{l, 1}\right) r_{t, 1}\right\}+\left(4 \mathrm{rl} 0^{3}\right.\right. \\
& +9 \pi\left\{r_{t, 0}\left[4 r_{l, 0}^{3}+3 r_{l, 1} r_{l, 0}^{2}+2 r_{l, 0} r_{l, 1}^{2}+r_{l, 1}^{3}+\left(r_{t, 1}+4 r_{l, 0}\right) r_{t, 0}^{2}\right]\right. \\
& +\left[r_{l, 0}^{3}+2 r_{l, 1} r_{l, 0}^{2}+3 r_{l, 0}\left(r_{l, 1}^{2}+r_{t, 0}^{2}\right)+2 r_{l, 1}\left(2 r_{l, 1}^{2}+r_{t, 0}^{2}\right)\right] r_{t, 1} \\
& \left.+\left(2 r_{l, 0}+3 r_{l, 1}\right) r_{t, 1}^{2} r_{t, 0}+\left(r_{l, 0}+4 r_{l, 1}\right) r_{t, 1}^{3}\right\} \\
& +12 a_{1}^{2} \pi\left[r_{l, 0}\left(2 r_{t, 0}+3 r_{t, 1}\right)+3 r_{l, 1}\left(r_{t, 0}+4 r_{t, 1}\right)\right] \\
& +8 a_{0}\left[8 r_{t, 0}\left(6 r_{l, 0}^{2}+3 r_{l, 0} r_{l, 1}+r_{l, 1}^{2}+3 r_{t, 0}^{2}\right)+2\left(6 r_{l, 0}^{2}+8 r_{l, 0} r_{l, 1}+6 r_{l, 1}^{2}+9 r_{t, 0}^{2}\right) r_{t, 1}\right. \\
& +12 r_{t, 0} r_{t, 1}^{2}+6 r_{t, 1}^{3}+6 a_{1}^{2}\left(2 r_{t, 0}+3 r_{t, 1}\right) \\
& \left.+3 \pi a_{1}\left(3 r_{l, 0} r_{t, 0}+2 r_{l, 1} r_{t, 0}+2 r_{l, 0} r_{t, 1}+3 r_{l, 1} r_{t, 1}\right)\right] \\
& +16 a_{1}\left\{4 r_{l, 0} r_{l, 1}\left(2 r_{t, 0}+3 r_{t, 1}\right)+r_{l, 0}^{2}\left(6 r_{t, 0}+4 r_{t, 1}\right)\right. \\
& \left.\left.+3\left[r_{t, 0}^{3}+2 r_{t, 0}^{2} r_{t, 1}+3 r_{t, 1}^{2} r_{t, 0}+4 r_{t, 1}^{3}+2 r_{l, 1}^{2}\left(r_{t, 0}+4 r_{t, 1}\right)\right]\right\}\right\} \text {. }
\end{aligned}
$$


In the current study, based on the model, we provide data for the mass-inertial parameters of the male upper limb and compare our results with those available from the literature. In order to do that, for the whole upper limb we use a realization of the model within a CAD system - SolidWorks. The computer-generated model has been carefully verified.

Table 2: Moments of inertia of the body segments through the center of mass $\left(\mathrm{kg} . \mathrm{cm}^{2}\right)$ for males, compared with the already performed analytical analysis.

\begin{tabular}{|l|l|l|l|l|l|l|}
\hline SEGMENT & \multicolumn{2}{|l|}{ ANALYTICAL CALCULATIONS } & \multicolumn{2}{l|}{ PRESENT DATA using 3D (GMM) } \\
\hline & Ixx & \multicolumn{1}{|l}{ Iyy } & \multicolumn{1}{|l|}{ Izz } & \multicolumn{1}{l|}{ Ixx } & Iyy & Izz \\
\hline UPPER ARM & 156.466 & 161.414 & 19.1882 & 156.47462 & 161.42413 & 19.19043 \\
\hline LOWER ARM & 42.8028 & 44.4078 & 5.05819 & 42.81349 & 44.42104 & 5.06232 \\
\hline HAND & 4.37 & 4.37 & 4.37 & 4.3691 & 4.3691 & 4.3691 \\
\hline
\end{tabular}

We have compared the data obtained within the computer model with those derived from the analytical results for the segment. We have found excellent agreement between these data for the different segments of the upper limb. The program reproduces segment-by-segment data about volume, mass, center of mass and moments of inertia - see Table 2 . That gives us confidence that this model could be used to calculate these characteristics for the whole upper limb. After determining them on the basis of the computer model, we compared our model results with the results available in literature for mass, volume and center of mass of the whole human upper limb for persons belonging to the same or similar type. The data are given in Tables 3, 4 and 5, respectively. The inspection shows a reasonably good agreement between our results and those previously reported in the literature. Data for the moments of inertia of the whole upper limb are reported in Table 6 . The authors are not aware of other such data for the moments of inertia of the upper limb - almost all data available in the literature are for different segments, not for limbs as a whole. A visualization of the principal CAD model realization of the human upper limb within the SolidWorks media is shown in Fig. 2.

Table 3: Mass of the whole male's upper limb (kg).

\begin{tabular}{|l|c|c|c|}
\hline & Min & Max & Mean \\
\hline Braune and Fisher (right) & 3.52 & 4.95 & 4.01 \\
\hline Braune and Fisherc(left) & 3.48 & 4.79 & 3.99 \\
\hline Chandler et al.(right) & 2.65 & 4.42 & 3.36 \\
\hline Chandler et al.(left) & 2.71 & 4.32 & 3.35 \\
\hline Clauser et al. & 2.65 & 4.18 & 3.22 \\
\hline Dempster (right) & 2.12 & 3.95 & 2.97 \\
\hline Dempster (left) & 2.13 & 3.89 & 2.87 \\
\hline Drillis and Contini & - & - & 4.38 \\
\hline Harless (right) & 2.63 & 4.47 & 3.39 \\
\hline Harless (left) & 2.45 & 3.77 & 2.94 \\
\hline Zatsiorsky and Seluyanov & - & - & 3.60 \\
\hline Our data & - & - & 3.25 \\
\hline
\end{tabular}

Table 4: Volume of the whole male's upper limb $\left(10^{-3} \mathrm{~m}^{3}\right)$.

\begin{tabular}{|l|c|c|c|}
\hline & Min & Max & Mean \\
\hline Chandler et al.(right) & 2.60 & 4.36 & 3.28 \\
\hline Chandler et al.(left) & 2.63 & 4.27 & 3.25 \\
\hline Clauser et al. & 2.38 & 3.96 & 2.98 \\
\hline Drillis and Contini & 3.51 & 4.58 & 3.97 \\
\hline Harless (right) & 2.40 & 4.09 & 3.25 \\
\hline Harless (left) & 2.22 & 2.39 & 2.31 \\
\hline Our data & - & - & 3.02 \\
\hline
\end{tabular}


Table 5: Location of the center of mass of the whole male's upper limb measured from the proximal end $(\mathrm{cm})$.

\begin{tabular}{|l|c|c|c|}
\hline & Min & Max & Mean \\
\hline Chandler et al. & 29.2 & 37.7 & 32.0 \\
\hline Drillis and Contini & - & - & 25.3 \\
\hline Our data & - & - & 28.5 \\
\hline
\end{tabular}

Table 6: Predicted moments of inertia of the body segments through the center of mass $\left(\mathrm{kg} . \mathrm{cm}^{2}\right)$ for males. The data are obtained by 3D-GMM computer analysis.

\begin{tabular}{|l|l|l|c|}
\hline Assembly & \multicolumn{1}{|c|}{ Ixx } & Iyy & Izz \\
\hline Upper + lower arm & 62.670388 & 63.326094 & 24.25276 \\
\hline Upper + lower arm + hand & 1220.06762 & 1226.62468 & 28.62192 \\
\hline
\end{tabular}

The calculation procedure of 3D GMM is oriented to application in medicine (orthopaedics and traumatology, orthotics and prosthesis design), anthropomorphic and rehabilitation robotics, ergonomics, sport, etc. The proposed model can predict data for the inertial parameters of a given male individual provided, if the corresponding easily measurable geometrical data for this particular individual are known.

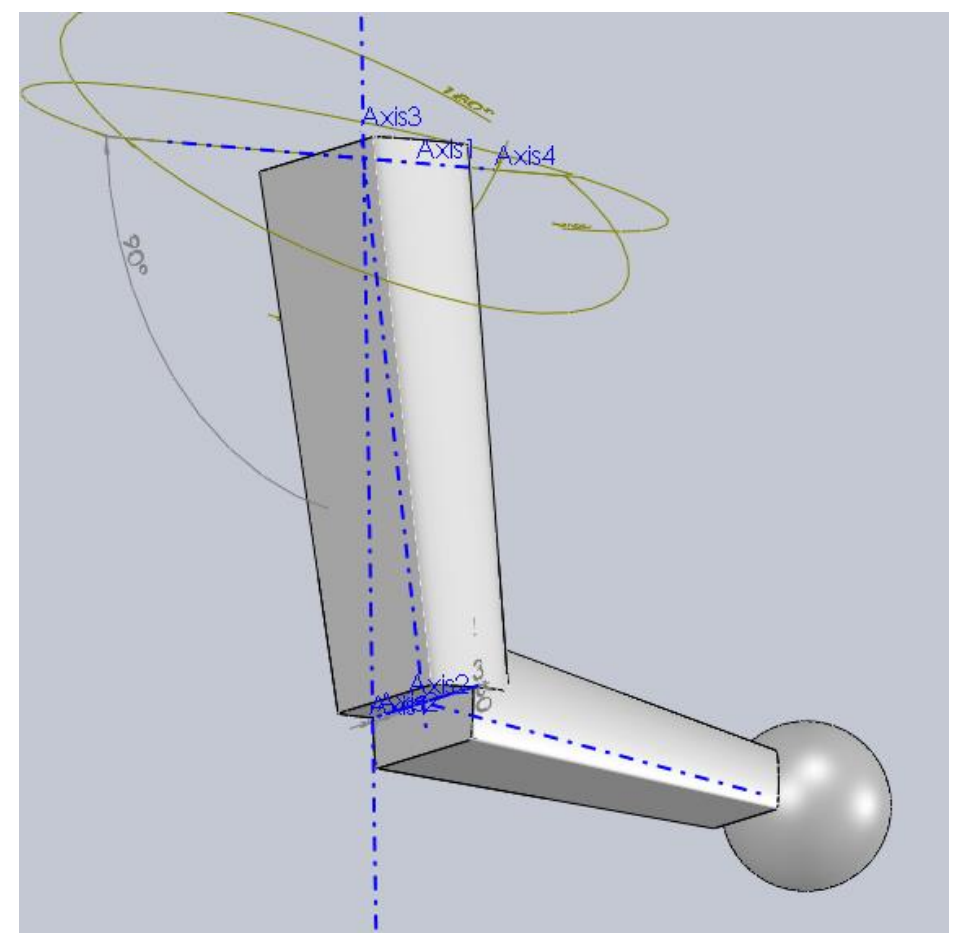

Fig. 2: SolidWorks media realization of the human upper limb model.

The computer generated model allows also to study the mass-inertial characteristics of the upper limb in any reasonable position and, thus, to study dynamics, and to predict characteristics which devices aimed to help a disabled person shall have in order to assist in performing a given specific motion.

\section{Conclusions}

In this paper, a new approach for determination of the human upper limb mass-inertial characteristics is presented by using the 3D geometrical mathematical modelling analysis approach. These results, as compared with the analytically calculates ones, demonstrate a very good concord with each other. There is also a good agreement with previously published data in the available literature. That is why authors believe that the presented approach is a qualitative 
contribution to the technique of determination of the mass moments of inertia, presented so far, that can be in addition reliably and with confidence used to determine the mass inertial characteristics of the whole upper limb and to study different position of the limb, as well as its dynamics, etc.

\section{Acknowledgements}

The financial support by the Bulgarian National Science Fund: Contract DN-07/5 "Study of anthropometric and massinertial characteristics of the Bulgarian men and women via mathematical models of the human body" is gratefully acknowledged.

\section{References}

[1] G. Nikolova, Y. Toshev, "Estimation of male and female body segment parameters of the Bulgarian population, using a 16-segmental mathematical model," Journal of Biomechanics, vol. 40, pp. 3700-3707, 2007.

[2] G. Nikolova, "Anthropometric measurements and model evaluation of mass-inertial parameters of the human upper and lower extremities," Medicon 2010, IFMBE Proceedings 29, pp. 574-577, 2010.

[3] G. Nikolova, "Segmental Parameters of Bulgarian man within modified known human body model," Journal of Theoretical and Applied Mechanics, vol. 40, no. 3, pp. 101-116, 2010.

[4] G. Nikolova, "Segmental parameters of Bulgarian woman within modified known human body model," Journal of Theoretical and Applied Mechanics, vol. 40, no. 4, pp. 73-80, 2010.

[5] E. Harless, "Die statishen momente der menschlichen gliedmassen," Abh.d.math.phys., Cl.d.konigl.Bayer, Akad.d.Wiss., vol. 8, 1860.

[6] W. Braune and O. Fischer, "The center of gravity of the human body as related to the equipment of the german infantryman," Treat. of the Math-Phys., vol. 26, 1889.

[7] W. T. Dempster, "Space requirements of the seated operator," WADC Technical Report 55-159, Wright-Patterson Air Force Base, Ohio, 1955.

[8] C. E. Clauser, J. T. McConville, J. W. Young, "Weight, volume, and center of mass of segments of the human body," Technical Report AMRL-TR-69-70, Wright-Patterson Air Force Base, Ohio, 1969.

[9] R. F. Chandler, C. E. Clauser, J. T. McConville, H. M. Reynolds, J. W. Young, "Investigation of inertial properties of the human body," Technical Report AMRL-TR-74-137, Wright-Patterson Air Force Base, Ohio, 1975.

[10] R. Drillis, R. Contini, "Body segment parameters," Technical Report 1166.03, New York University, School of Engineering and Science, New York, 1966.

[11] V. M. Zatsiorsky, V. N. Seluyanov, "The mass and inertia characteristics of the main segments of the human body," In: Matsui, H., Kobayashi, K. (Eds.), Biomechanics VIII-B. Human Kinetics, Champaign, IL, pp. 1152-1159, 1983.

[12] E. P. Hanavan, "A mathematical model of the human body," AMRLTR-64-102, Aerospace Medical Research Laboratories, Wright-Patterson Air Force Base, Ohio, 1964.

[13] Y.-H. Kwon, Kwon3D motion analysis, 1999, [Online]. Available: http://kwon3d.com

[14] Y. Yordanov, A. Nacheva, S. Tornjova, N. Kondova, B. Dimitrova, D. Topalova, "Anthropology of the Bulgarian population at the end of the $20^{\text {th }}$ century (30-40 years old persons)," Professor Marin Drinov Academic Publishinh House, Sofia, Bulgaria, 2006.

[15] J. Bjornstrup, "Estimation of human body segment parameters - statistical analysis of results from prior investigations," Technical Report ISSN 0906-6233, LIA 96-7, Laboratory of Image analysis, Institute of Electronic Systems, Aalborg University, Denmark, 1996. 